\title{
Divided attention impairs detection of simple visual features
}

\author{
Amelia H. Harrison ${ }^{1,2}$, Sam Ling ${ }^{2,3}$, and Joshua J. Foster ${ }^{2,3}$ \\ ${ }^{1}$ Department of Psychological and Brain Sciences, University of California, Santa Barbara \\ ${ }^{2}$ Department of Psychological and Brain Sciences, Boston University \\ ${ }^{3}$ Center for Systems Neuroscience, Boston University
}

Corresponding author:

Joshua J. Foster

Department of Psychological and Brain Sciences

Boston University

677 Beacon Street

Boston, MA 02215

Email: jifoster@bu.edu

Conflicts of interest: None

Author contributions: All authors designed the study. AHH and JJF performed the experiments, analyzed the data, and drafted the manuscript. All authors revised the manuscript, and approved the final version for submission.

Acknowledgements: This work was supported by R01 EY28163 to SL, and a Boston University Center for Systems Neuroscience Postdoctoral Fellowship to JJF. 


\begin{abstract}
Covert spatial attention allows us to prioritize processing at relevant locations. There is substantial evidence that perception is poorer when attention is distributed across multiple locations than when attention is focused on a single location. However, recent work suggests that may not always be the case: divided attention does not appear to impair detection of simple visual features that are represented in primary visual cortex. Here, we re-examined this possibility. In two experiments, observers detected a simple target (a vertical Gabor), and we manipulated whether attention was focused at one location (focal-cue condition) or distributed across two locations (distributed-cue condition). In Experiment 1, targets could appear independently at each location. Thus, observers needed to judge target presence for each location separately in the distributed-cue condition. Under these conditions, we found a robust cost of dividing attention. In this experiment, the cost of dividing attention could reflect either a limit in perceptual processing or a limit in decision making. Therefore, in Experiment 2, we simplified the task to more directly test whether dividing attention impairs perceptual processing of the target. Specifically, only one target could appear on each trial, such that observers made the exact same decision in both conditions ("was a target present?"). Here, we found a marginal cost of dividing attention on performance, that was weaker than the cost in Experiment 1. Together, our results suggest that divided attention does impair detection of simple visual features, but that this cost is primarily due to limits in post-perceptual decision making.
\end{abstract}

Keywords: attention; divided attention; detection; capacity limits 


\section{Introduction}

Our capacity to process sensory information is limited. Thus, we must prioritize the processing of relevant information to the detriment of other information. Covert spatial attention plays a central role in this effort, allowing us to prioritize processing at relevant locations without moving our eyes. Decades of research has shown that covert attention improves perception, such that observers can resolve finer details and detect fainter signals at attended locations than at unattended locations (for review, see Carrasco, 2011). A substantial body of work has shown that dividing covert attention across multiple locations impairs perceptual performance. These studies found that performance is worse when covert attention is spread over multiple locations in the visual field, compared to when attention is focused at a single, cued location (e.g. Itthipuripat et al., 2014; Ling \& Carrasco, 2006; McMains \& Somers, 2005; Pestilli et al., 2011).

Recent evidence, however, suggests that there may be an exception to this rule: dividing attention may not impair detection of simple visual features (Moreland et al., 2020; Scharff et al., 2011; White et al., 2017, 2018). White et al. (2017) noted that studies that have reported costs of dividing attention have used tasks that involve higher-order demands. For example, many studies of divided attention have required observers to detect or discriminate conjunctions of features (e.g., letters or objects), which are encoded in higher visual areas, where attention has a substantial impact on neural responses (for review, see Maunsell, 2015), while other studies have used tasks that place demands on working memory because successive stimuli must be compared across short delays (e.g. Itthipuripat et al., 2014; Pestilli et al., 2011). White et al. (2017) reasoned that divided attention may not 
impair detection of simple features that are encoded in primary visual cortex (V1), such as orientation, because attention effects are small or absent in V1 (Chen \& Seidemann, 2012; Moran \& Desimone, 1985; Yoshor et al., 2007). For example, Chen and Seidemann (2012) had monkeys perform a detection task under focal- and distributed-attention conditions, and found that dividing attention did not reduce the amplitude of stimulus-evoked response in V1. Indeed, White et al. (2017) found that detection of orientation targets was not impaired when attention was divided compared to when attention is focused at a single location (for similar results, see Moreland et al., 2020; Scharff et al., 2011; White et al., 2018; but see Chen \& Seidemann, 2012).

There are two reasons that these results are surprising. First, although there is evidence that attentional modulation of neural responses in V1 is small or absent (Chen \& Seidemann, 2012; White et al., 2017; Yoshor et al., 2007), other work has contested the view that attention effects in V1 are negligible (e.g. Hembrook-Short et al., 2017, 2019; Briggs et al., 2013). Second, even if divided attention does not attenuate sensory representations in $\mathrm{V} 1$, divided attention might nevertheless impair detection of simple features via modulation of representations in higher visual areas, or due to limits in postperceptual stages of processes. Thus, even if dividing attention does not degrade sensory representations in early visual cortex, it might still lead to poorer detection of simple visual features due to limits in later stages of processing.

The finding that divided attention does not impair detection of simple features (Moreland et al., 2020; Scharff et al., 2011; White et al., 2017, 2018) warrants further investigation because it challenges the widely embraced notion that dividing attention impairs 
perceptual performance in general. In this study, we re-examined whether detection of simple features is impaired when attention is divided across multiple locations. In two experiments, we asked observers to detect vertical target gratings, overlaid over a horizontal pedestal (Figure 1). We modeled this detection task after two recent studies of divided attention (Chen \& Seidemann, 2012; White et al., 2017). Across blocks of the task, we manipulated whether observers focused on one of two locations (focal-cue condition) or distributed across both locations (distributed-cue condition).

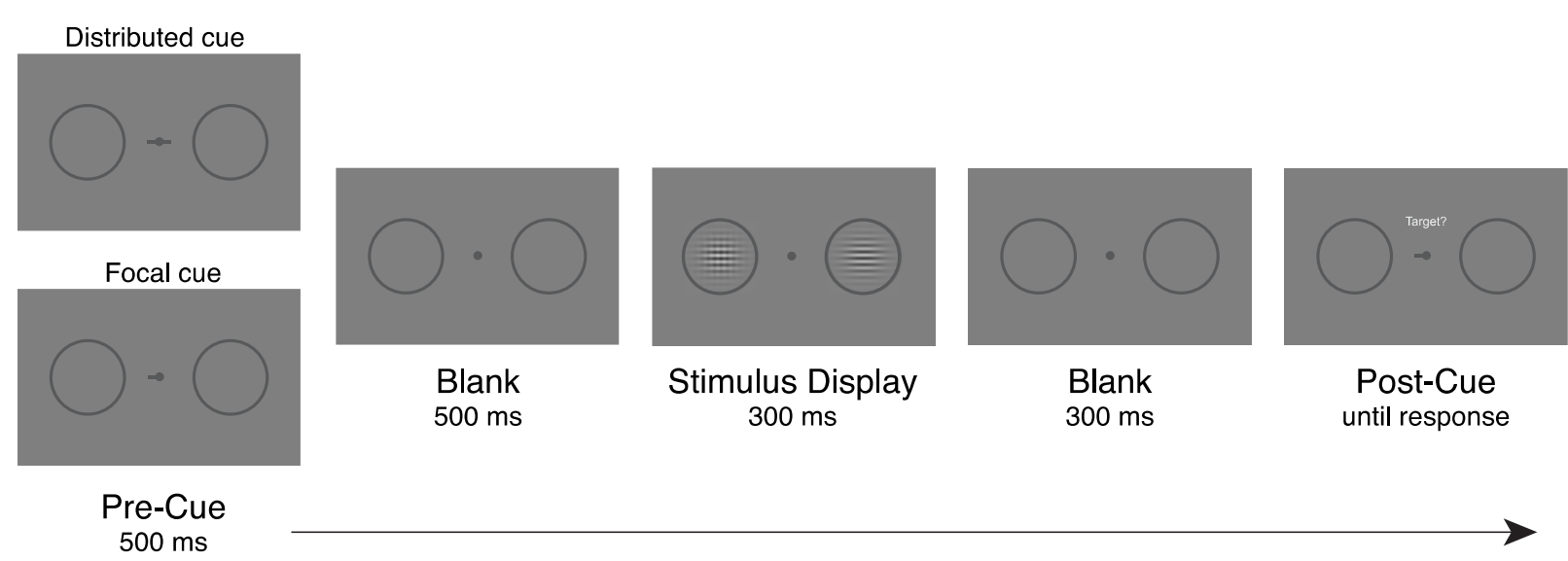

Figure 1. Example trial sequence. Observers were asked to report whether a vertical target (present in the left aperture in this example) appeared on the side indicated by a post-cue. In the distributed-cue condition, the pre-cue was spatially uninformative. In the focal-cue condition, one side was cued in advance. In Experiment 1, targets could appear in each aperture independently. In Experiment 2, only one target could appear on any given trial. Cue validity in the focal cue-condition was 90\% in Experiment 1 and 100\% in Experiment 2. The size of the cue and fixation point are exaggerated here for clarity.

In Experiment 1, targets could appear independently at each location (with 50\% probability), as was the case in recent studies that have found no effect of dividing attention on detection of simple features (Moreland et al., 2020; White et al., 2017, 2018). Therefore, in the distributed-cue condition, observers needed to judge whether a target was present at each location separately, and were then promoted to report whether a target was present at 
the post-cued location. In the focal-cue condition, the post-cued location matched pre-cued location with $89 \%$ validity, and mismatched on a small fraction (11\%) of invalidly cued trials. Invalidly cued trials allowed us to verify that observers used the pre-cue to direct attention (manifesting as better performance for validly cued trials than for invalidly cued trials), in case we found no difference in performance between the focal-cue and distributed-cue conditions. In this experiment, a cost of divided attention might reflect poorer sensory coding of the target when attention is distributed, but could also reflect a limit in decision-related stages of processing when observers must make multiple decisions.

In Experiment 2, we simplified the task: just one target could appear on each trial (also with $50 \%$ probability), and observers judged whether a target was present or absent, rather than making that same judgement for each location separately. The target (when present) appeared always at the cued location in the focal-cue condition or could appear at either location in the distributed-cue condition. Thus, observers made the exact same decision ("was a target present?") in both conditions, with the only difference being whether the targets location was known in advance of the stimulus display. We reasoned that if distributed attention impairs sensory coding of simple-featured targets, then perceptual sensitivity should be lower in the distributed-cue condition than in the focal cue condition. However, if we instead observe no cost of dividing attention in Experiment 2, that result would suggest any cost observed in our first experiment reflected a limit in decision-related stages of processing.

\section{Methods}




\section{Data Availability and Pre-registration}

All data and analysis code will be made available on Open Science Framework at the time of publication. We pre-registered the methods and analysis plan for Experiment 2 on Open Science Framework before we began data collection (see osf.io/6np4v/).

\section{Participants}

Participants were recruited from the Boston University community. All participants were at least 18 years old, reported normal or corrected-to-normal visual acuity, and provided informed consent according to procedures approved by the Boston University Institutional Review Board. Because the experiment was run during the COVID-19 pandemic, participants were screened for COVID-19 risk factors prior to enrollment in the study, and we excluded individuals with health conditions that increased their risk of developing a severe case of COVID-19 in accordance with Boston University guidelines at the time of data collection. Participants were screened for COVID-19 symptoms the day before testing and upon arrival at the lab. Participants were compensated $\$ 12 /$ hour for participating in the study.

\section{Experiment 1}

For Experiment 1, our target sample size was 10 participants, following White et al. (2018). Fourteen volunteers participated in the study (6 males and 8 females, mean age $=$ 20.1 years, SD = 1.2). Three participants were excluded from the final sample for the following reasons: one participant withdrew from the study; we did not bring one participant back for the second session of the study because we were unable to obtain adequate eye tracking data for that participant; and we did not bring another participant back for the 
second session of the study because they broke fixation on more than $25 \%$ of trials in the first session. The final sample was 11 participants ( 3 males and 8 females, mean age $=20.0$ years, $S D=1.3)$. We exceeded our target sample size of 10 participants by one because the final participant was scheduled before reaching our target sample size.

\section{Experiment 2}

For Experiment 2, our target sample size was 12 participants This sample size was determined based on the effect size (Cohen's $d_{z}$, see Statistical Analysis) found for the cost of divided attention in Experiment 1 of approximately 1.5. Anticipating that the effect may be smaller in Experiment 2, we carried out a power analysis, performed using G*Power 3.1 (Faul et al., 2009), which revealed that approximately 11 participants would be needed to detect an effect size of 1.0 with $90 \%$ power. Thus, we set a target sample of 12 participants so that we could counterbalance block order across participants (see pre-registration: osf.io/6np4v/). Sixteen volunteers participated in the study (2 males and 14 females, mean age $=21.4$ years, $S D=1.8)$. Four participants were excluded from the sample for the following reasons: one participants withdrew from the study; we did not bring one participant back for the second session of the study because we were unable to obtain adequate eye tracking data for that participant; we did not bring another participant back for the second session of the study because they broke fixation on more than $25 \%$ of trials in the first session; and one participant was excluded because task performance after staircasing was at ceiling (accuracy of 98.7\%). The final sample was 12 participants (2 males and 10 females, mean age $=21.3, S D=1.8$. 


\section{Apparatus and Stimuli}

Stimuli were generated using MATLAB (The MathWorks) and the Psychophysics Toolbox (Brainard, 1997; Pelli, 1997) on a PC running Ubuntu Version 16.04 LTS. Participants viewed stimuli on a gamma-corrected LCD monitor (Display++, Cambridge Research Systems Limited; $100 \mathrm{~Hz}$ refresh rate, $1440 \times 1080$ resolution). The full range of luminance was resolved to 14 bits (i.e., 16,384 luminance levels), which enabled precise control of the contrast of the low-contrast target Gabor patches. Participants were seated comfortably with their chin on a padded chin rest at a viewing distance of $135 \mathrm{~cm}$. Stimuli were presented against a mid-grey background $\left(108 \mathrm{~cd} / \mathrm{m}^{2}\right)$. There were no additional light sources in the room. Participants made their responses on a standard keyboard. Participants wore a face mask during the study in accordance with Boston University's COVID-19 research guidelines.

\section{Task Procedures}

\section{Experiment 1}

Figure 1 shows a schematic of the detection task that participants performed. Participants fixated a dark gray dot $\left(0.16^{\circ}\right.$ in diameter, $\left.88 \mathrm{~cd} / \mathrm{m}^{2}\right)$ at the center of the screen and began a trial by pressing the spacebar. At the start of each trial, circular apertures appeared to the left and right of fixation $\left(6.0^{\circ}\right.$ in diameter, $0.2^{\circ}$ thick, centered $5.66^{\circ}$ horizontally from fixation, same luminance as the fixation dot), which marked the locations at which each stimulus would appear. These apertures remained on-screen for the duration of the trial. After a jittered interval between 300 and 500 ms, a pre-cue was presented for 500 ms. For the distributed-cue condition, this was composed of two small horizontal bars $\left(0.18^{\circ}\right.$ 
long, $0.12^{\circ}$ thick) that extended from fixation, pointing to either side of the screen. Thus, the pre-cue provided no information about which side the participants would be tested on in the distributed-cue condition. In the focal-cue condition, just one bar appeared, either pointing to the left or right side of the screen; this bar indicated the likely side that the participant would be tested on. The pre-cue was valid on $89 \%$ of trials. For the $500 \mathrm{~ms}$ after the precue, only the fixation dot and apertures remained on-screen. Next, the stimulus display was presented for 300 ms. In this display, a horizontally oriented Gabor patch (2 cycles per degree, Gaussian envelope with standard deviation of $1^{\circ}, 20 \%$ Michelson contrast) was presented in each of the apertures. These horizontal Gabor patches served as pedestals for potential targets, which were vertically oriented Gabor patches. On each trial, there was a $50 \%$ chance that a target was present on each side, determined independently. Only the fixation dot and apertures remained on-screen for $300 \mathrm{~ms}$ after the stimulus display before a response screen was presented. Finally, the participant reported whether or not a target was present on the side indicated by a post-cue. On invalidly cued trials, the post-cue was black rather than grey to make it obvious to the participant that they were being tested on the uncued side because these trials were uncommon. Participants responded using the number pad of the keyboard (' 1 ' = target present, ' 2 ' = target absent). The participant's response was displayed above the fixation dot ("yes" or "no" for target present or target absent, respectively), and the participant could correct their response if they pressed the wrong key. There was no time limit for inputting a response. The participant confirmed their response by pressing the spacebar, and feedback was then presented for $300 \mathrm{~ms}$ : If the response was correct, the fixation dot flashed white; if the response was incorrect, the fixation dot flashed 
black and the word "Incorrect" was displayed above the fixation dot in black text. We instructed participants to maintain fixation and to avoid blinking for the duration of each trial (from the time the pre-cue was triggered to appear until the time the response screen was displayed). Participants were free to blink or move their eyes in between trials. Trials during which blinks or breaks from fixation were detected with an eye tracker were aborted, and responses were not collected on these trials (see Eye Tracking).

Following White and colleagues (White et al., 2017, 2018), we manipulated the cue condition (focal cue vs. distributed cue) across blocks. The pre-cue conditions (focal-cue and distributed-cue) were alternated across blocks, with the order counterbalanced across participants. Each block of the distributed-cue condition included 64 trials, with the left and right stimuli probed equally often, such that participants needed to divide attention between the two stimuli. Each block of the focal-cue condition included 72 trials (64 validly cued trials and 8 invalidly cued trials, such that cue validity was approximately $89 \%)$. Participants completed two sessions, each comprising 12 blocks, six focal-cue blocks and six distributedcue blocks. The left side was cued in half of the focal-cued blocks, and the right side was cued in the other half (c.f. Moreland et al., 2019; White et al., 2017, 2018). In the first session, which took approximately 2 hours, participants were familiarized with the task, and we adjusted task difficulty by staircasing the contrast of the vertical Gabor targets (see Staircase Procedure). In the second session, which took approximately 1.5 hours, the contrast of the targets on each side of the display were set based on performance in the first session. All analyses were performed on data acquired in the second session.

\section{Experiment 2}


Experiment 2 was identical to Experiment 1 except for three changes. First, and most substantially, just one target could appear on each trial (with 50\% probability), and participants judged whether a target was present or absent, rather than making that same judgement for each location separately. Second, for efficiency, we did not include invalidly cued trials in focal-cued blocks, which comprised 64 validly cued trials. Thus, the target always appeared on the cued side in the focal-cued condition (i.e. the pre-cue was $100 \%$ valid). Third, we increased the sample size based on a power analysis performed on the basis of Experiment 1 (see Participants, Experiment 2).

\section{Staircase Procedure}

In the first session of each experiment, we adjusted contrast of the vertical targets independently for the left and right apertures, for both the focal-cue and distributed-cue conditions. Thus, we ran four independent staircases. We used a weighted up/down procedure to adjust target contrast: after a correct response, target contrast decreased by 5\%; after an incorrect response, target contrast increased by $17.6 \%$. This procedure was intended to held accuracy fixed at approximately $76 \%$. However, in practice, accuracy was generally higher than $76 \%$ in the second session of the experiment because performance continued to improve with experience. The contrast of the targets in the second session (for both the focal-cue and distributed-cue conditions) were determined on the basis of the performance in the focal-cued conditions in the staircase session. We included the distributed-cue conditions in the staircase session solely to ensure that participants had equal practice with the focal-cued and distributed-cued conditions prior to the second session of the experiment. We set the contrast of the targets for each side of the display to 
the mean value of the staircase across the last 80 trials of the staircase. For some participants in Experiment 1, we averaged across more trials to determine the contrast values if the staircase had clearly reached an asymptote sooner. In Experiment 1, the mean target contrast after staircasing was $1.21 \%(S D=0.28)$ for the left aperture and $1.16 \%(S D$ $=0.22)$ for the right aperture. In Experiment 2, the mean target contrast was 1.09\% (SD = $0.30)$ for the left aperture and $1.14 \%(S D=0.26)$ for the right aperture.

\section{Eye Tracking}

We monitored gaze position at $1000 \mathrm{~Hz}$ with a desk-mounted infrared eye tracker (Eyelink 1000 Plus, SR Research), which provides spatial resolution of $0.01^{\circ}$ of visual angle, and average accuracy of $0.25-0.50^{\circ}$ of visual angle. In both experiments, trials were aborted if participants broke fixation or blinked to ensure that participants maintained steady fixation. We calibrated the eye tracker throughout the session as needed. We operationalized a break in fixation as the participant's gaze exceeding a specified distance from the fixation dot. This threshold was initially set to $1^{\circ}$ from fixation. A threshold of $1^{\circ}$ was too stringent for some participants, for whom noise in the eye tracking data resulted in too many false alarms. We increased the threshold for these participants. The threshold was never larger than $1.5^{\circ}$. If the participant broke fixation during a trial, the text "Eye movement" appeared above the fixation point, and a black line extended from the fixation point to the recorded gaze position to indicate where the participant had moved their eyes. If the participant blinked during a trial, the word 'Blink' was immediately displayed above the fixation point. The participant was free to initiate the next trial after this feedback. We excluded participants for whom more than $25 \%$ of trials were aborted due to blinks or eye movements (see Participants). In Experiment 
1 , an average of $8.7 \%(S D=3.6)$ trials were aborted for participants in the final sample. In Experiment 2, and an average of $6.5 \%(S D=3.2)$ trials were aborted in for participants in the final sample.

\section{Statistical Analysis}

For each observer, we calculated sensitivity to vertical targets, $d^{\prime}=\mathrm{z}$ (hit rate) $-\mathrm{z}$ (false alarm rate), for each condition separately. We used an alpha level of .05 for statistical tests. Statistical results for directional hypotheses are noted as one tailed and non-directional hypotheses are noted as two tailed. We calculated effect sizes for paired-samples t-tests as Cohen's dz:

$$
d_{z}=\frac{t}{\sqrt{n}}
$$

where $t$ is the t-statistic and $n$ is the sample size, and for independent-samples t-tests as Cohen's ds:

$$
d_{s}=t \sqrt{\frac{1}{n_{1}}+\frac{1}{n_{2}}}
$$

where $t$ is the t-statistic, and $n_{1}$ and $n_{2}$ are the number of observations in each sample (see Lakens, 2013). We used the BayesFactor Package (version 0.9.12; Morey \& Rouder, 2018)) for R to calculate Jeffreys-Zellner-Siow (JZS) Bayes factor from t-statistics $(r$-scale $=0.7071)$.

\section{Results}

\section{Detection Performance}

\section{Experiment 1}

In Experiment 1, we tested whether divided attention impairs detection of vertical targets (Figure 1). Figure 2a shows sensitivity (d') separately for the valid focal-cue, 
distributed-cue, and invalid focal-cue conditions. Sensitivity was significantly higher in the focal-cue condition when the cue was valid $(M=2.12, S D=0.67)$ than when it was invalid $(M=0.73, S D=0.61), t(10)=6.74, p<.001$, Cohen's $d_{z}=2.03, B F_{10}=1033.58$ (one-tailed test), confirming that observers took advantage of the pre-cue to orient attention to the cued location. Next, we tested whether dividing attention impairs target detection by comparing sensitivity for the valid focal-cue and distributed-cue conditions. We found that sensitivity was higher in the focal-cue condition $(\mathrm{M}=2.12, \mathrm{SD}=0.67)$ than in the distributed-cue condition $(M=1.59, S D=0.53), t(10)=4.88, p<.001$, Cohen's $d_{z}=1.47, B F_{10}=117.90$ (one-tailed test). The Bayes factor indicates very strong evidence for a cost of dividing attention. Thus, observers were considerably poorer at detecting vertical targets when covert attention was distributed across two locations than when attention was focused at one location.
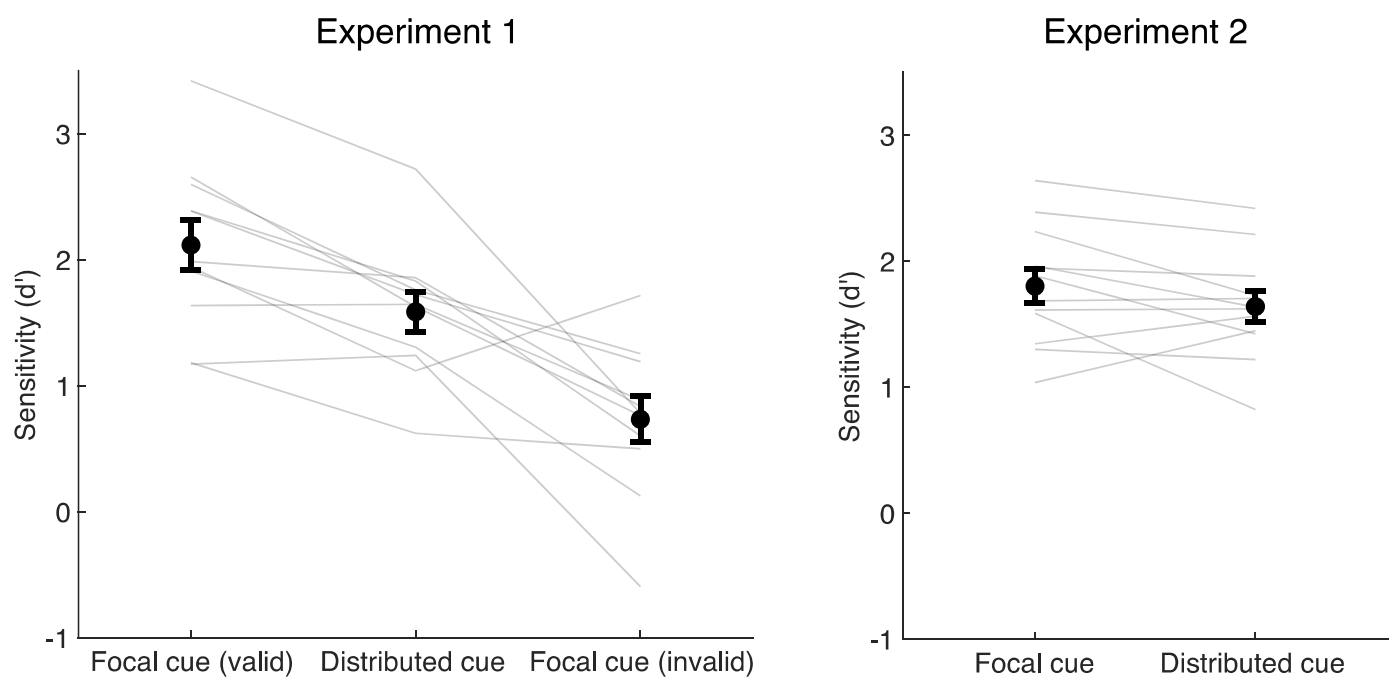

Figure 2. Target detection sensitivity (d') for each experiment. Black circles and error bars show the mean \pm 1 SEM across participants. Gray lines show data for each participant.

\section{Experiment 2}


In Experiment 2, observers performed the same detection task as in Experiment 1 with one major difference: only one target could appear on any given trial. Therefore, in the distributed-cue condition, observers judged whether a target was present or absent regardless of location, rather than making that same judgement for each location separately. For efficiency, we did not include invalidly cued trials in this experiment, having already established that observers used the pre-cue in Experiment 1. Figure 2b shows sensitivity in the focal-cue and distributed-cue conditions. As in Experiment 1, sensitivity was higher in the focal-cue condition $(M=1.80, S D=0.47)$ than in the distributed-cue condition $(M=1.64$, $S D=0.42)$, however, this modest difference was not significant, $t(11)=1.73, p=0.11$, Cohen's $d_{z}=0.50, B F_{10}=1.71$ (one-tailed test). The Bayes factor indicates weak evidence for a cost of dividing attention in this experiment. Figure 3 shows the cost of dividing attention on sensitivity (valid focal-cue - distributed cue) in each experiment. An independent-samples t-test confirmed that the cost of dividing attention was significantly larger in Experiment 1 ( $M$ $=0.53, S D=0.36)$ than in Experiment $2(M=0.16, S D=0.32), t(21)=2.58, p=.0175$, Cohen's $d_{s}=1.08, B F_{10}=3.44$. Thus, although the evidence for a cost of dividing attention was ambiguous in Experiment 2, this cost was smaller in Experiment 2 than in Experiment 1. 


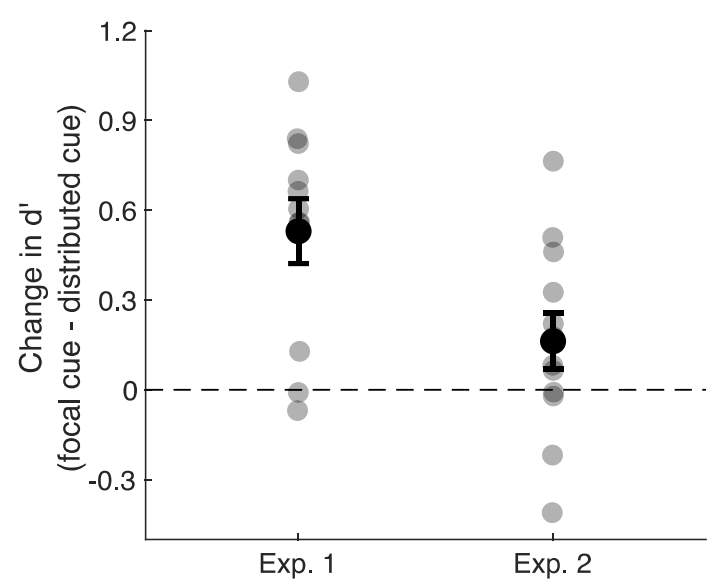

Figure 3. Cost of dividing attention on sensitivity (d') for each experiment. Black circles and error bars shown the mean \pm 1 SEM across participants. Gray circles show data for each participant.

\section{Eye Movement Controls}

In both experiments, we used a gaze-contingent procedure to abort trials with blinks or eye movements to ensure that observers maintained steady fixation throughout each trial (see Methods, Eye Tracking). However, small residual biases in eye position toward the cued location might nevertheless remain if observers consistently made sub-threshold eye movements that were not detected by the gaze-continent procedure. Figure 3 shows the mean gaze position across time as a function of cued location. The first two participants in Experiment 1 are not included in this analysis because the eye tracking data were not saved due to a technical error, although the gaze-contingent procedure did operate for these participants. In both experiments, mean horizontal gaze position during the stimulus display varied by less than $0.1^{\circ}$ across pre-cued locations. Thus, bias in eye position toward the precued location cannot account for differences in detection performance between conditions focal-cue and distributed-cue conditions. 

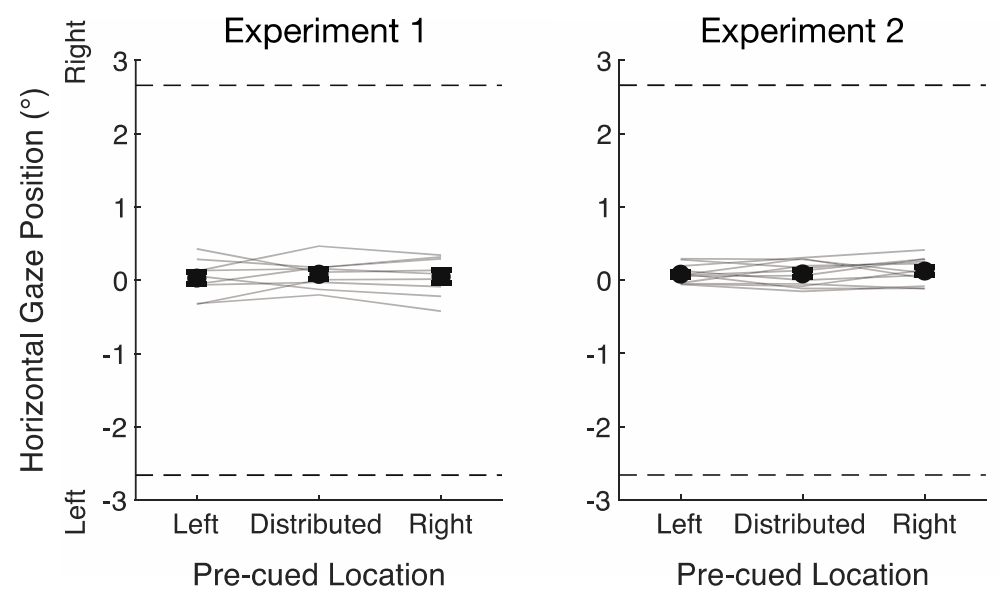

Figure 4. Eye movement controls. Each plot shows the average horizontal gaze position relative to the fixation dot during the 300-ms stimulus display as a function of pre-cued location. Black circles and error bars show the mean \pm 1 SEM across subjects (the error bars are smaller than the circle in some cases). Gray lines show data for each participant. Dashed lines at the top and bottom of each plot mark the inner edge of the stimulus apertures at $2.66^{\circ}$ of visual angle.

\section{Discussion}

In two experiments, we tested whether dividing covert attention across multiple locations impairs the detection of simple visual features. In Experiment 1, targets appeared independently at each of two locations. Therefore, in the focal-cue condition, observers could make a single judgement about the presence of a target at the cued location, whereas in the distributed-cue condition, observers needed to judge whether a target was present at each location separately. As a result, poorer performance in the distributed-cue condition could reflect a cost in decision-related stages of processing. In this experiment, we found a robust cost of dividing attention on detection performance. In Experiment 2, we simplified the task. In this experiment, only one target was present on any given trial. In both the focal-cue and distributed-cue conditions, observers judged whether a target was present or absent, regardless of its location, such that only difference between the conditions was whether the target location was cued in advance. In this experiment, the evidence for a cost of dividing 
attention was equivocal. The Bayes factor suggested that this experiment did not provide compelling evidence for or against a cost of dividing attention, and the cost of dividing attention in this experiment was significantly smaller than the cost in Experiment 1. Together, our results suggest that dividing covert attention does indeed impair detection of simple visual features, but that this cost is largely due to a limit in decision-related stages of processing.

Our results are at odds with recent studies that have found that dividing attention does not impair detection of simple visual features (Moreland et al., 2019; White et al., 2017; White et al., 2019). In these studies, as was the case in our first experiment, targets could appear independently at each of the stimulus locations. For example, White et al. (2017) presented observers with two streams of dynamic noise and asked observers to detect brief target Gabors presented within this noise (White et al.'s Experiment 2). In the focal-cue condition, observers were pre-cued to the relevant stimulus, allowing them to focus exclusively on the cued location. In the distributed-cue condition, observers needed to judge target present at each location, and report whether a target was present on the side indicated by a post-cue. White et al. (2017) found no cost in detection performance due to divided attention (also see Moreland et al., 2019; White et al., 2018). It is somewhat surprising that these studies found no cost of divided attention. Even if divided attention does not degrade sensory coding of the stimuli, detection performance may be worse in the distributedattention due to limits in post-perceptual processing because observers must make two independent decisions in the distributed-cue condition, rather than a single decision in the focal-cue condition. Thus, no cost in detection performance suggests that observers can 
process two stimuli in parallel, at both sensory and decision-related stages of processing. Our results tell a different story. Our first experiment was closely modeled after these recent studies, but unlike these studies, we found a robust cost of dividing attention on detection performance. In our second experiment, we did not find a reliable cost of dividing attention when we simplified the task demands so that observers did not need to make two independent decisions in the distributed-cue condition. This finding suggests that the robust cost of divided attention in our first experiment reflects a limit in post-perceptual, decisionrelated stages of processing.

It is unclear why we observed a clear cost of divided attention in our first experiment while other similar studies (e.g. White et al., 2017) have not. We think the discrepancy is unlikely to be due to stimulus parameters because we modeled our stimuli closely after those used by White et al.'s (2017) Experiment 1. One factor that may be important to consider is the participants themselves. We recruited volunteers that had little previous experience performing psychophysical task, and it is not entirely clear how our participants compared in this respect with those in previous studies (e.g. White et al., 2017).

Although our results are incompatible with those of White et al. (2017), our results are consistent with other studies. For example, Chen and Seidemann (2012) had monkeys perform detect a vertical target over a horizontal pedestal, much like the detection task used in our experiments and by White et al. (2017) in their Experiment 1. Chen and Seidemann (2012) found that detection performance was poorer in the distributed-cue condition than in the focal-cue condition. Interestingly, however, Chen and Seidemann also found that divided attention did not attenuate stimulus-evoked responses in $\mathrm{V} 1$, which led them to conclude, 
like us, that the cost in behavioral performance reflected limits in later stages of processing. It must be noted that there are some important differences to consider between Chen and Seidemann's experiment and our own. In their experiment, as in our Experiment 2, only one target could be present on each trial in the distributed-cue condition. Thus, at first glance, Chen and Seidemann found a cost of divided attention under conditions where we did not. However, importantly, in their experiment, the animals reported target presence by making a saccade to the target's location. Therefore, their task involved a location-discrimination component that our Experiment 2 did not. This additional task requirement might explain why Chen and Seidemann found a cost of dividing attention while we did not in Experiment 2. Further work is needed to delineate the precise boundary condition between our experiments and Chen and Seidemann's. Nevertheless, Chen and Seidemann's results are broadly consistent with our conclusion that the cost of divided attention for detection of simple visual features is primarily reflects limits in later stages of processing.

\section{Conclusions}

Dividing attention impairs perception. However, recent studies have suggested that dividing covert attention may not impair detection of very simple visual features that are coded in early sensory cortex (Moreland et al., 2020; White et al., 2017, 2018). In this study, we re-visited this possibility. In contrast to these recent studies, we found that divided attention produced a robust cost in detection of simple orientation targets. Interestingly, the cost of divided attention was only significant in our first experiment, where post-perceptual stages of processing could limit performance, but not in our second experiment, which provided a purer test of whether dividing attention impairs perceptual processing specifically. 
Our results suggest that dividing attention impairs the detection of simple features due to limits in decision-related stages of processing. 


\section{References}

Brainard, D. H. (1997). The Psychophysics Toolbox. Spatial Vision, 10(4), 433-436.

Briggs, F., Mangun, G. R., \& Usrey, W. M. (2013). Attention enhances synaptic efficacy and the signal-to-noise ratio in neural circuits. Nature, 499(7459), 476-480.

https://doi.org/10.1038/nature12276

Carrasco, M. (2011). Visual attention: The past 25 years. Vision Research, 51(13), 14841525. https://doi.org/10.1016/j.visres.2011.04.012

Chen, Y., \& Seidemann, E. (2012). Attentional modulations related to spatial gating but not to allocation of limited resources in primate V1. Neuron, 74(3), 557-566.

https://doi.org/10.1016/j.neuron.2012.03.033

Faul, F., Erdfelder, E., Buchner, A., \& Lang, A.-G. (2009). Statistical power analyses using G*Power 3.1: Tests for correlation and regression analyses. Behavior Research Methods, 41(4), 1149-1160. https://doi.org/10.3758/BRM.41.4.1149

Hembrook-Short, J. R., Mock, V. L., \& Briggs, F. (2017). Attentional modulation of neuronal activity depends on neuronal feature selectivity. Current Biology: $C B, 27(13), 1878-$ 1887.e5. https://doi.org/10.1016/j.cub.2017.05.080

Hembrook-Short, J. R., Mock, V. L., Usrey, W. M., \& Briggs, F. (2019). Attention enhances the efficacy of communication in V1 local circuits. Journal of Neuroscience, 39(6), 10661076. https://doi.org/10.1523/JNEUROSCI.2164-18.2018 Itthipuripat, S., Ester, E. F., Deering, S., \& Serences, J. T. (2014). Sensory gain outperforms efficient readout mechanisms in predicting attention-related improvements in 
behavior. Journal of Neuroscience, 34(40), 13384-13398.

https://doi.org/10.1523/JNEUROSCI.2277-14.2014

Lakens, D. (2013). Calculating and reporting effect sizes to facilitate cumulative science: A practical primer for t-tests and ANOVAs. Frontiers in Psychology, 4, 863.

https://doi.org/10.3389/fpsyg.2013.00863

Ling, S., \& Carrasco, M. (2006). Sustained and transient covert attention enhance the signal via different contrast response functions. Vision Research, 46(8-9), 1210-1220. https://doi.org/10.1016/j.visres.2005.05.008

Maunsell, J. H. R. (2015). Neuronal mechanisms of visual attention. Annual Review of Vision Science, 1(1), 373-391. https://doi.org/10.1146/annurev-vision-082114-035431

McMains, S. A., \& Somers, D. C. (2005). Processing efficiency of divided spatial attention mechanisms in human visual cortex. Journal of Neuroscience, 25(41), 9444-9448. https://doi.org/10.1523/JNEUROSCI.2647-05.2005 Moran, J., \& Desimone, R. (1985). Selective attention gates visual processing in the extrastriate cortex. Science, 229(4715), 782-784.

https://doi.org/10.1126/science.4023713

Moreland, J. C., Palmer, J., \& Boynton, G. M. (2020). Set-size effects in change detection depend on failures of retrieval and/or comparison and not on perception, encoding or storage. 2020.01.19.911867. bioRxiv. https://doi.org/10.1101/2020.01.19.911867 Morey, R. D., Rouder, J. N., Jamil, T., Urbanek, S., Forner, K., \& Ly, A. (2018). Package "BayesFactor." https://cran.r-project.org/web/packages/BayesFactor/ 
Pelli, D. G. (1997). The VideoToolbox software for visual psychophysics: Transforming numbers into movies. Spatial Vision, 10(4), 437-442.

Pestilli, F., Carrasco, M., Heeger, D. J., \& Gardner, J. L. (2011). Attentional enhancement via selection and pooling of early sensory responses in human visual cortex. Neuron, 72(5), 832-846. https://doi.org/10.1016/j.neuron.2011.09.025

Scharff, A., Palmer, J., \& Moore, C. M. (2011). Extending the simultaneous-sequential paradigm to measure perceptual capacity for features and words. Journal of Experimental Psychology: Human Perception and Performance, 37(3), 813-833.

https://doi.org/10.1037/a0021440

White, A. L., Palmer, J., \& Boynton, G. M. (2018). Evidence of serial processing in visual word recognition. Psychological Science, 29(7), 1062-1071.

https://doi.org/10.1177/0956797617751898

White, A. L., Runeson, E., Palmer, J., Ernst, Z. R., \& Boynton, G. M. (2017). Evidence for unlimited capacity processing of simple features in visual cortex. Journal of Vision, 17(6). https://doi.org/10.1167/17.6.19

Yoshor, D., Ghose, G. M., Bosking, W. H., Sun, P., \& Maunsell, J. H. R. (2007). Spatial attention does not strongly modulate neuronal responses in early human visual cortex. Journal of Neuroscience, 27(48), 13205-13209.

https://doi.org/10.1523/JNEUROSCI.2944-07.2007 